\title{
ESTRESSORES EM ADOLESCENTES SOBREVIVENTES DE CÂNCER
}

\author{
STRESSORS IN TEENAGER CANCER SURVIVORS
}

\author{
Michele Daiane Birck ${ }^{\mathrm{a}^{*}}$, Áderson Luiz Costa Junior ${ }^{\mathrm{b}^{*}}$ \\ amicheledaianepsi@yahoo.com.br, baderson@unb.br \\ *Universidade de Brasília - Brasília (DF), Brasil
}

Data de recebimento do artigo: 11/10/2013

Data de aceite do artigo: 08/05/2014

\section{RESUMO}

Objetivo: Descrever e analisar os principais estressores relatados pelos sobreviventes de câncer infantil sob o ponto de vista destes e de seus pais/cuidadores. Materiais e método: Participaram do estudo 8 adolescentes sobreviventes de câncer vinculados à Associação Brasileira de Assistência às Famílias de Crianças Portadoras de Câncer e Hemopatias (Abrace), no Distrito Federal, bem como seus cuidadores (8 mães, 2 pais e 1 avó). Como critérios de inclusão, os adolescentes deveriam ser sobreviventes de câncer primário (independentemente do tipo), tendo finalizado o tratamento hospitalar, em função da cura da neoplasia, entre cinco e doze anos antes da pesquisa, ter idade mínima de 12 e máxima de 18 anos no momento da coleta e poderiam ser de ambos os sexos. Os instrumentos utilizados foram o Youth Self Report (YSR), para avaliação dos estressores nos jovens sobreviventes, e o Child Behavior Checklist $(C B C L)$, respondido pelos pais/cuidadores. Resultados: Os principais estressores dos sobreviventes estáo relacionados a retraimento e depressão, bem como a problemas de atençáo. Conclusáo: Os dados de comparação entre respostas dos pais e dos filhos sugerem que, apesar de serem bons informantes de problemas emocionais e comportamentais de seus filhos, os pais podem subestimar a capacidade dos sobreviventes em lidar com sintomas de ansiedade e depressão.

Palavras-chave: câncer infantil; psico-oncologia pediátrica; sobrevivência; estressores.

\section{ABSTRACT}

Objective: To describe and analyze the main stressors reported by survivors of childhood cancer from their point of view of and their parents/caregivers. Method: The participants of this study were 8 adolescents survivors of cancer linked to the Brazilian Association for Assistance to Families of Children with Cancer and Hematological (ABRACE) in the Federal District, as well as their caregivers (8 mothers, 2 fathers and 1 grandmother). As criteria for inclusion, were included adolescents who were survivors of primary cancer (regardless of type), of both sexes, aged between 12 and 18 years old at the time of collection, who had concluded the hospital treatment due to the healing neoplasia five to 12 years before the research. The instruments used were the Youth Self Report (YSR) for evaluation of the stressors in young survivors and the Child Behavior Checklist (CBCL), answered by the parents/caregivers. Results: The main stressors of survivors are related to withdrawal and depression, as well as attention issues. Conclusion: The data comparing answers of parents and children suggest that, despite the parents being good informants of emotional and behavioral problems of their children, they may underestimate the ability of survivors to cope with symptoms of anxiety and depression.

Keywords: Childhood cancer; psycho-oncology pediatric; survival; stressors. 
Apesar de se usar a terminologia "sobrevivente de câncer" há algumas décadas, não há consenso sobre a sua definição. O National Coalition for Cancer Survivors define que sobrevivente é qualquer pessoa que tenha sido diagnosticada com câncer, do momento do diagnóstico até o fim da vida ${ }^{1}$. O National Cancer Institute amplia a definição, incluindo a família e os cuidadores do paciente ${ }^{2}$. Little, Paul, Jordens e Sayers restringem esse conceito à pessoa que vive em qualquer fase após o fim do tratamento e que se encontra aparentemente livre da doença ${ }^{3}$. Já a Childhood Cancer Foundation considera uma criança tecnicamente curada se os exames não mostram sinais da doença cinco anos depois de encerrado o tratamento ${ }^{4}$. Por sua vez, Meadows propóe que o paciente de câncer pediátrico seja definido como alguém que sobreviveu ao menos cinco anos desde a última evidência da doença e que concluiu o tratamento há pelo menos dois anos 5 . Barakat, Alderfer e Kazak adotam a perspectiva de sobrevivente como o indivíduo que está fora de tratamento há, no mínimo, um ano ${ }^{6}$.

Pode-se, assim, verificar uma falta de consenso que permita explicitar claramente o conceito de sobrevivente. Numa perspectiva biomédica, o conceito é definido num espaço temporal (dois, cinco ou dez anos) ou analisado como uma fase (após o fim da terapêutica), ou como um resultado da sobrevida (curado). Embora a barreira dos cinco anos não tenha suporte científico universal, continua tendo grande importância psicológica, pois, no senso comum, perpetua a ideia de que é a fronteira para o ingresso na fase da cura ${ }^{7}$.

A partir de uma revisão de literatura, Patenaude e Kupst salientam que resultados de estudos em psico -oncologia pediátrica têm mostrado a existência de um pequeno, mas notável grupo de crianças sobreviventes e membros familiares que não enfrentam bem ou que têm muitas dificuldades pessoais, familiares e sociais ${ }^{8}$. Segundo os autores, esses sobreviventes de câncer pediátrico apresentam problemas como insucesso escolar, dificuldades de conseguir emprego, piora ou diminuição dos relacionamentos sociais, problemas com autoconceito, autoestima ou identidade. Trabalhos têm examinado a prevalência de sintomas de estresse pós-traumático, e descobriu-se que esses sintomas de moderados a graves estão presentes em torno de $5 \%$ a $20 \%$ dos sobreviventes?.

Estressores refletem preocupaçóes práticas, questóes sociais, falta de habilidades, preocupaçóes religiosas ou espirituais e problemas médicos e físicos que ex-pacientes e seus familiares enfrentam. Em geral, os estressores dos sobreviventes de câncer incluem medo da recorrência e da morte, pensamentos intrusivos sobre o câncer, relacionamentos alterados, problemas com operadoras de plano de saúde e sintomas físicos do tratamento ${ }^{10}$. As repercussóes físicas e emocionais de um tratamento de câncer muitas vezes comprometem o bem-estar emocional e prejudicam a qualidade de vida global dos ex-pacientes, o que demanda esforços adicionais, cognitivos e emocionais, deles e de seus familiares.

Estudos voltados a compreender e identificar a repercussão psicossocial do tratamento sobre os sobreviventes abordam questóes relacionadas a autoimagem, ajustamento psicossocial pós-tratamento, desempenho escolar, sentimentos de discriminação e expectativas da família, em especial dos pais e irmãos, ante a situação vivenciada pelo familiar ${ }^{11,12}$.

Pesquisadores e clínicos têm observado que o tipo e o grau de estressores podem variar de acordo com a fase da sobrevivência ao câncer, a qual pode ser um tempo de incertezas em função do fim das intervençôes médicas hospitalares e do contato cada vez menor com os profissionais da saúde de quem os pacientes e seus familiares recebiam informação técnica e apoio ampliado.

O objetivo deste estudo é descrever e analisar os principais estressores relatados pelos sobreviventes de câncer infantil em seus contextos de desenvolvimento e comparar os estressores na percepçáo dos adolescentes e de seus pais/cuidadores.

\section{Materiais e método}

Este estudo teve delineamento transversal, e a sua natureza é descritiva, uma vez que o objetivo era sistematizar o conhecimento acerca da sobrevivência em oncologia pediátrica, com destaque aos estressores.

\section{Participantes}

Foram entrevistados 8 adolescentes sobreviventes de câncer infantil (todos, por coincidência, do sexo masculino), bem como seus cuidadores ( 8 mães, 2 pais e 1 avó). A coleta dos dados ocorreu na residência dos participantes, durante o mês de janeiro de 2013. Seu nome e seus dados de contato haviam sido fornecidos pela Abrace, uma instituição filantrópica, declarada de utilidade pública, criada, em 1986, por um grupo de pais cujas crianças faziam tratamento de câncer. Os selecionados para este estudo, residentes no Distrito Federal (DF) ou em localidades do estado de Goiás identificadas como do entorno do DF, concordaram em participar.

O diagnóstico de cada paciente bem como dados familiares sociodemográficos foram extraídos dos relatos da família. Como critérios de inclusão, os adolescentes deveriam ser sobreviventes de câncer primário (independentemente do tipo), tendo finalizado o tratamento hospitalar, em funçáo da cura da neoplasia, entre cinco e doze anos antes da pesquisa (caracterizando-se como 
sobreviventes a longo prazo), ter idade mínima de $12 \mathrm{e}$ máxima de 18 anos no momento da coleta e poderiam ser de ambos os sexos. Como critério de exclusão para convite à participaçáo, os adolescentes não poderiam ser sobreviventes de câncer secundário ou metastático nem apresentar dificuldades em funçóes cognitivas que impedissem a compreensão de instruções verbais e a resposta verbal às questóes formuladas.

\section{Instrumentos}

\section{Questionário Sociodemográfico}

Um questionário foi desenvolvido para este estudo e aplicado aos familiares a fim de coletar dados sociodemográficos da família e dados clínicos do sobrevivente. Foram coletados dados de identificação dos entrevistados que incluíam escolaridade, informaçóes acerca da doença, da saúde e dos comportamentos gerais dos sobreviventes e dados de renda familiar.

\section{Inventário de Comportamentos para os Jovens}

Foi utilizado o Inventário de comportamentos autorreferidos para jovens de 11 a 18 anos - versão brasileira do Youth Self Report for Ages 11-18 (YSR/11-18), proposta por Abreu, Bordin e Paula em 2001 ${ }^{13}$. Trata-se de um questionário que visa rastrear problemas emocionais e/ ou comportamentais em crianças e adolescentes de $11 \mathrm{a}$ 18 anos a partir de seus próprios pontos de vista.

O Youth Self Report é derivado do Child Behavior Checklist (CBCL) e foi traduzido para o Brasil por Abreu, Bordin e Paula, em 2001, e validado em estudo brasileiro por Rocha ${ }^{15}$. O YSR e o CBCL foram desenvolvidos a partir do início da década de 1960, nos Estados Unidos. O YSR é um questionário de autoavaliação para jovens entre 11 e 18 anos, composto por uma descrição simples de comportamentos problemáticos de crianças e adolescentes, que devem assinalar 0 (zero) se a afirmação for falsa, 1 (um) se a afirmação for mais ou menos verdadeira ou 2 (dois) se a afirmação for bastante verdadeira, tendo em conta os últimos seis meses de vida. As questóes do YSR foram elaboradas de forma a obter a percepção do jovem sobre si mesmo e em relação às suas competências ou dificuldades individuais ou grupais.

A pesquisadora do presente estudo aplicou a versão completa do Youth SelfReport a seus participantes sobreviventes de câncer, porém a primeira parte do inventário bem como os itens de comportamentos socialmente desejáveis da segunda parte não serão analisados, visto que não constituem os objetivos deste estudo. Também, na segunda parte do instrumento, dos itens totais de problemas emocionais/comportamentais em jovens, Rocha apresentou apenas 89 com carga fatorial significativa ( $\mathrm{p}$ $<0,01)$ em seu estudo no Brasil ${ }^{15}$; dessa forma, optou-se por analisar somente as respostas referentes aos 89 itens, conforme a Tabela 1.

\section{Inventário de Comportamentos para os Pais}

Foi utilizado o Inventário de comportamentos para crianças e adolescentes de 6 a 18 anos - versão brasileira do Child Behavior Checklist for Ages 6-18 (CBCL/6-18), traduzida por Bordin, Paula e Duarte em 20014.

O Child Behavior Checklist foi elaborado pelo psiquiatra norte-americano Thomas M. Achenbach e visa obter, de maneira padronizada, o relato dos pais sobre os comportamentos de seus filhos. O CBCL/6-18 é a versão para os cuidadores do YSR/11-18. No Brasil, Bordin, Mari e Caiero realizaram um estudo de validação preliminar com a versão anterior do $C B C L$, a partir do qual concluíram que o instrumento é válido para avaliar a população brasileira, visto que apresenta alto índice de correlação com a avaliação feita por um psiquiatra que não teve acesso aos dados do inventário $^{16,17}$. Atualmente, Emerich, Rocha e Silvares estão realizando uma investigação com a versão mais recente do $C B C L$ para obter índices de validade e normas para o instrumento ${ }^{18,19}$.

As respostas aos 89 itens do YSR e ao CBCL foram analisadas por meio do Microsoft Office Word e do Microsoft Office Excel'. A Tabela 1 apresenta as oito

Tabela 1: Escalas-síndromes do YSR/11-18 e do CBCL/6-18 e seus itens de avaliação.

\begin{tabular}{lc}
\hline Escalas-síndromes & Itens de avaliaçáo correspondentes \\
\hline Ansiedade/depressão & $14,29,30,31,32,33,35,45,50,52,71,91,112$ \\
Retraimento/depressão & $42,65,69,75,102,103,111$ \\
Queixas somáticas & $47,51,54,56 \mathrm{a}, 56 \mathrm{~b}, 56 \mathrm{c}, 56 \mathrm{~d}, 56 \mathrm{e}, 56 \mathrm{f}, 56 \mathrm{~g}$ \\
Violação de regras & $26,39,43,63,67,72,81,82,90,96,101,105$ \\
Comportamento agressivo & $11,12,25,27,34,36,38,48,62,64,79$ \\
Problemas de sociabilidade & $1,8,10,13,17,41,61$ \\
Problemas de atençáo & $3,16,19,20,21,22,23,37,57,68,86,87,89,94,95,97,104$ \\
Problemas com o pensamento & $9,18,40,46,58,66,70,76,83,84,85,100$ \\
\hline
\end{tabular}


escalas-síndromes do inventário de comportamentos para os jovens e do inventário de comportamentos para os pais e seus respectivos itens de avaliação.

O presente estudo utilizou-se dos instrumentos YSR e $C B C L$ para avaliar estressores em sobreviventes por compreender que os itens de problemas emocionais e comportamentais, de uma maneira geral, referem-se a preocupaçôes práticas, falta de habilidades, questóes sociais, preocupaçóes espirituais ou religiosas.

\section{Cuidados Éticos}

Para assegurar o cumprimento de normas éticas na condução de pesquisas com seres humanos, constantes na Resolução no 196/1996 do Conselho Nacional de Saúde, o projeto de pesquisa foi submetido à apreciação do Comitê de Ética em Pesquisa da Faculdade de Ciências da Saúde da Universidade de Brasília, credenciado à Comissão Nacional de Ética em Pesquisa (Conep), tendo sido aprovado em 18 de setembro de 2012 sob o protocolo no 079/2012. A participaçáo foi voluntária, e a inclusão no estudo ocorreu mediante a assinatura do Termo de Consentimento Livre e Esclarecimento (TCLE) pelos adolescentes e pelos pais/ cuidadores, no momento da visita à residência.

\section{Resultados}

\section{Análise do Inventário de Comportamentos dos Sobreviventes}

Os resultados do Youth Self Report mostraram que os maiores estressores dos sobreviventes estão relacionados a queixas de retraimento/depressão $(M=0,52)$, com destaque também para problemas de atenção ( $M$ $=0,44)$. As outras escalas do instrumento e suas respectivas médias foram, em ordem decrescente: ansiedade/ depressâo $(M=0,38)$, comportamento agressivo $(M=$ $0,34)$, problemas de sociabilidade $(M=0,32)$, problemas com o pensamento $(M=0,27)$, violação de regras $(\mathrm{M}=0,22)$ e, por último, queixas somáticas $(\mathrm{M}=0,09)$.

Com relação à escala retraimento/depressão, a pontuação dos itens foi, em gradação decrescente: "Sou muito tímido" (31\%); "Sou reservado, fechado, não conto minhas coisas para ninguém" (27,6\%); "Recusome a falar" (17,2\%); "Prefiro ficar sozinho a ficar na companhia dos outros" (13,8\%); "Não tenho muita energia" (6,9\%); e "Sou infeliz, triste ou deprimido" $(3,4 \%)$. O item "Sou retraído, não me relaciono com os outros" não obteve pontuação.
$\mathrm{Na}$ escala de problemas de atenção, o item mais pontuado foi: "Sou agitado" (40\%). "Ajo sem parar para pensar" obteve $16 \%$ das respostas, e "Comporto-me de modo infantil, como se tivesse menos idade" obteve $12 \%$. Os itens "Não vou bem na escola", "Tenho dificuldade para me concentrar", "Sinto-me confuso" e "Fico no mundo da lua, sonhando acordado" obtiveram $8 \%$ das respostas cada.

$\mathrm{Na}$ escala de ansiedade/depressão, o item que mais pontuou foi: "Sou apreensivo, aflito ou ansioso demais" $(17,5 \%)$, seguido de "Sou muito preocupado", "Fico sem jeito na frente dos outros com facilidade" (cada um com 15\%) e "Tenho medo de certos animais, situaçóes ou lugares" (10\%). Os itens "Penso em me matar", "Sinto-me desvalorizado, inferior" e "Tenho medo da escola" não tiveram nenhuma pontuação.

$\mathrm{Na}$ escala de comportamento agressivo, o item que mais pontuou foi: "Sou esquentado" (15,2\%), seguido de "Argumento muito" e "Sou desconfiado" (cada um com 13\%). "Destruo minhas próprias coisas", "Destruo coisas dos outros" e "Entro em muitas brigas" não tiveram nenhuma pontuação.

Com relaçáo à escala de problemas de sociabilidade, os itens mais pontuados foram: "Prefiro conviver com adolescentes mais novos" e "Tenho ciúmes dos outros" (cada um com 17,8\%). "Sou dependente demais dos adultos" obteve 14,3\%. "Machuco-me sem querer frequentemente" e "Sou desastrado, desajeitado" obtiveram 10,7\% cada um. Os itens "Acho que os outros me perseguem" e "As crianças ou adolescentes não gostam de mim" não obtiveram pontuação.

$\mathrm{Na}$ escala de problemas com o pensamento, houve destaque para o item "Não consigo tirar certos pensamentos da cabeça" $(30,7 \%)$. Os itens "Tento me machucar de propósito ou já tentei me matar" e "Escuto sons ou vozes que as pessoas acham que não existem" não tiveram pontuaçáo.

Com relação à escala de violação de regras, o item mais pontuado foi: "Xingo ou falo palavrōes" (28,6\%). Os outros itens não tiveram pontuaçóes importantes, ou não tiveram nenhuma pontuação.

Por último, na escala de queixas somáticas, houve destaque para o item "Sinto-me cansado demais sem motivo" (42,8\%). Os outros itens da escala foram muito pouco pontuados ou não obtiveram pontuação.

\section{Comparações entre as Respostas do Inventário de Comportamentos dos Sobreviventes com a de seus Cuidadores}

As respostas do autorrelato dos jovens de problemas emocionais/comportamentais do Youth Self Report foram comparadas com as respostas dos pais/cuidadores 
ao Child Behavior Checklist, a respeito dos mesmos problemas dos filhos.

$\mathrm{Na}$ soma total de respostas dos instrumentos, os pais relataram mais problemas comportamentais/emocionais $(\mathrm{n}=231)$ nos jovens do que os jovens autorrelataram $(n=222)$. A diferença mais importante entre as pontuaçóes de pais e filhos ocorreu na escala de ansiedade/depressão, cuja resposta dos pais $(\mathrm{n}=54)$ foi maior que o autorrelato dos jovens $(\mathrm{n}=40)$. Constatou-se, no entanto, que a média total de respostas dos pais $(\mathrm{M}=$ $23,1)$ ficou relativamente próxima da média total de autorrelatos dos jovens $(\mathrm{M}=27,75)$ para as escalas-síndromes do YSR e do CBCL.

\section{Discussão}

Os resultados obtidos neste estudo apontam algumas informações relevantes à prática clínica dos profissionais de saúde que atuam na área da psico-oncologia pediátrica. Jovens curados de câncer entendem que algo grave ocorreu com eles, percebem a preocupação da família e dos outros ao seu redor e convivem, muitas vezes, com os efeitos tardios do tratamento em seu organismo. $\mathrm{O}$ estudo de Greenberg, Kazak e Meadows indicou que crianças e adolescentes sobreviventes de câncer com piores efeitos tardios do tratamento tiveram autoconceito total mais pobre, mais sintomas depressivos e um maior lócus de controle externo do que aqueles sobreviventes sem efeitos, ou com efeitos tardios leves ou moderados ${ }^{20}$.

Possivelmente, sintomas de retraimento/depressão estão relacionados à gravidade ou mesmo à visibilidade dos efeitos tardios nos sobreviventes. No presente estudo, em seis dos oito sobreviventes entrevistados, os efeitos do tratamento eram visíveis fisicamente. Outro estressor identificado no presente estudo está relacionado com problemas de atenção nos sobreviventes. Os jovens reportaram que se percebem agitados e que agem sem parar para pensar. Schultz et al. identificaram que pontuaçôes na depressão/ansiedade, no déficit de atenção e nos domínios antissociais foram significativamente elevados em adolescentes sobreviventes de câncer infantil tratados por leucemia ou tumores do sistema nervoso central, quando comparados com seus irmãos ${ }^{21}$. Além disso, os sobreviventes de neuroblastoma tiveram dificuldades na depressáo/ansiedade e domínios antissociais. Tratamentos com radiaçáo craniana e/ou metotrexato intratecal foram fatores de risco específicos para consequências sociais e comportamentais adversas.

Pesquisadores sugerem aumento da prevalência de problemas psicológicos em adolescentes sobreviventes de câncer infantil, também pelos desafios esperados desta fase da vida ${ }^{22}$. No presente estudo, os entrevistados são todos adolescentes, e algumas dificuldades comportamentais e cognitivas poderiam estar intensificadas, como, por exemplo, maior dificuldade de concentração.

As médias totais de respostas de problemas emocionais/comportamentais relatados pelos pais e autorrelatados pelos jovens foram relativamente próximas, sugerindo que os pais são bons informantes do estado emocional e comportamental de seus filhos. No entanto, uma diferença importante nas respostas entre pais e filhos apareceu neste trabalho. Os pais relataram mais queixas relacionadas à escala de ansiedade/depressão em seus filhos em comparação com as outras escalas. Esse fato sugere algumas possibilidades. Entre elas, um indicativo de ansiedade e depressão nos próprios familiares, as quais influenciam suas respostas. Outra possibilidade é que os filhos se sentem melhores, adotam formas de lidar com situações adversas da sobrevivência de uma forma melhor que seus pais conseguem identificar.

É importante destacar, como afirmam Araujo e Arraes, que, por um lado, sobreviventes de câncer podem se sentir aliviados e eufóricos com o término do tratamento, mas, por outro, podem se sentir inseguros sem a proteção do ambiente hospitalar e o suporte medicamentoso ${ }^{23}$. Além disso, podem perceber-se como vulneráveis. Esse fator tende a ser altamente ansiogênico tanto para o sobrevivente quanto para sua família. Essa afirmação vai ao encontro de Delella e Araujo ${ }^{24}$; em seu estudo, as autoras perceberam que crianças demonstraram estar melhor adaptadas à sobrevivência ao câncer do que seus pais. No entanto, as vivências associadas à experiência oncológica ainda se recobriam de significados negativos para todos.

\section{Conclusão}

Os serviços de saúde que atendem e acompanham os sobreviventes de câncer infantil podem valer-se das informaçóes deste estudo para investigarem junto ao paciente possíveis problemas emocionais e comportamentais que comprometem seu bem-estar e sua qualidade de vida. Muitas vezes, o comportamento reservado, fechado perante os outros, dificulta que o sobrevivente exponha o que sente e o que necessita. Não se sabe se não expóe porque prefere ser reservado, sente-se tímido no contato com o outro, pois é um adolescente e essa pode ser uma característica da própria fase do desenvolvimento; ou porque, de alguma forma, não se sente no direito de falar de suas queixas, pois deveria estar feliz por estar vivo.

Os sintomas de dificuldades comportamentais e sociais em sobreviventes podem estar relacionados com o câncer infantil e seu tratamento e justificam a avaliaçáo de uma equipe multidisciplinar, incluindo psicólogos com experiência na área de sobrevivência ao câncer infantil. 
Como terapias para o câncer infantil evoluem, os estudos de acompanhamento são importantes na avaliação do impacto de protocolos de tratamento em sobreviventes, bem como de resultados comportamentais e sociais.

O presente estudo trata de um tema ainda pouco investigado no Brasil. Sugere-se que estudos futuros comparem sobreviventes em curto prazo com sobreviventes em longo prazo com relação a estressores. Entre as limitaçóes identificadas, destaca-se a sua amostra pequena e composta exclusivamente de meninos. $\mathrm{O}$ estudo focou a descrição dos dados encontrados e entende que deve haver cautela com relação a generalizaçôes a outras populações de sobreviventes de câncer infantil.

\section{Referências}

1. National Coalition for Cancer Survivorship. Teamwork: the cancer patient's guide to talking with your doctor [Internet]. 2011. Disponível em: <http://www.canceradvocacy.org/ wp-content/uploads/2013/01/Teamwork.pdf>. Acesso em: 20 janeiro 2013.

2. National Cancer Institute. Supervivencia: la vida con cáncer y después del tratamiento [Internet]. 2004. Disponível em: $<$ http://www.cancer.gov/espanol/cancer/sobrellevar/supervivencia>. Acesso em: 03 fevereiro 2013.

3. Little M, Paul K, Jordens CFC, Sayers EJ. Survivorship and discourses of identity. Psycho-Oncology. 2002;11:170-8.

4. Childhood Cancer Foundation. Childhood cancer survivors: a practical guide to your future [Internet]. 2010. Disponível em: <http://www.candlelighters.ca/index. html>. Acesso em: 07 fevereiro 2013.

5. Meadows AT. Pediatric cancer survivors: past history and future challenges. Current Problems in Cancer. 2003;27:112-26.

6. Barakat LP, Alderfer MA, Kazak AE. Posttraumatic growth in adolescent survivors of cancer and their mothers and fathers. J Pediatr Psychol. 2006;31:413-9.

7. Pinto C, Ribeiro JLP. A qualidade de vida dos sobreviventes de cancro. Rev Port Saúde Pública. 2006;24:37-56.

8. Patenaude AF, Kupst MJ. Psychosocial functioning in pediatric cancer. J Pediatr Psychol. 2005;30:9-27.

9. Hobbie WL, Stuber M, Meeske K, Wissler K, Rourke M, Ruccione K, et al. Symptoms of posttraumatic stress in young adult survivors of childhood cancer. J Clin Oncol. 2000;18:4060-6.

10. Lauver DR, Connolly-Nelson K, Vang P. Stressors and coping strategies among female cancer survivors after treatments. Cancer Nurs. 2007;30:101-11.

11. Anders JC, Souza AIJ. Crianças e adolescentes sobreviventes ao câncer: desafios e possibilidades. Ciênc Cuid Saúde. 2009;8:131-7.

12. (Ki) Moore IM. Cancer in children. In Hayman LL, Mahon MM, Turner JR, editors. Chronic illness in children: an evidence-based approach. Nova York: Springer; 2002. p. 80-103.

13. Achenbach TM. Youth self-report for ages 11-18 (YSR/1118). Burlington: University of Vermont; 2001a. Versão brasileira Abreu SR, Bordin IAS, Paula CS. Inventário de comportamentos autorreferidos para jovens. São Paulo: Departamento de Psiquiatria, Universidade de São Paulo.

14. Achenbach TM. Child behavior checklist for ages 6-18 (CBCL/6-18). Burlington: University of Vermont; 2001b. Versão brasileira Bordin IAS, Paula CS, Duarte CS. Inventário de comportamentos para crianças e adolescentes. São Paulo: Departamento de Psiquiatria, Universidade de São Paulo.

15. Rocha MM. Evidências de validade do inventário de autoavaliação para adolescentes (YSR/2001) para a população brasileira [Tese]. São Paulo: Universidade de São Paulo. No prelo.

16. Bordin IAS, Mari JJ, Caieiro MF. Validação da versão brasileira do child behavior checklist (CBCL) (inventário de comportamentos da infância e adolescência): dados preliminares. Revista ABP-APAL. 1995;17:55-66.

17. Achenbach TM. Manual for the child behavior checklist and 1991 profile. Burlington: University of Vermont, Department for Psychiatry; 1991.

18. Emerich DR, Rocha MM, Silvares EFM. Estudo de validação preliminar do inventário dos comportamentos de crianças e adolescentes de 6 a 18 anos (CBCL/6-18), versão brasileira do Child Behavior Checklist [projeto de pesquisa submetido ao comitê de ética em pesquisa com seres humanos do Instituto de Psicologia da Universidade de São Paulo]; 2010.

19. Rocha MM, Ferrari RA, Silvares EFM. Padróes de concordância entre múltiplos informantes na avaliação dos problemas comportamentais de adolescentes: implicaçóes clínicas. Estud Pesqui Psicol. 2011;11:948-64.

20. Greenberg HS, Kazak AE, Meadows AT. Psychologic functioning in 8 to 16 year old cancer survivors and their parents. The Journal of Pediatrics. 1989 Mar;114(3):488-93.

21. Schultz KA, Ness KK, Whitton J, Recklitis C, Zebrack B, Robison LL, et al. Behavioral and social outcomes in adolescent survivors of childhood cancer: a report from the childhood cancer survivor study. J Clin Oncol. 2007 Aug 20;25(24):3649-56.

22. Krull KR, Huang S, Gurney JG, Klosky JL, Leisenring W, Termuhlen A, et al. Adolescent behavior and adult health status in childhood cancer survivors. Journal of Cancer Survivorship. 2010;4:210-7.

23. Araujo TCCF, Arraes AR. A sobrevivência em oncologia: uma vivência paradoxal. Psicol Ciênc Prof. 1998;18:2-9.

24. Delella LA, Araujo TCCF. Câncer na infância: uma investigação sobre a avaliação da desordem de estresse pós-traumático parental e a experiência da sobrevivência. Psicol Argum. 2002;20:43-8. 